\title{
LA THÉORIE DES PLAQUES ÉLASTIQUES PLANES*
}

\author{
PAR \\ JACQUES HADAMARD
}

L'équilibre des plaques élastiques planes et, plus particulièrement, la mise en équation de ce problème, ont fait l'objet de travaux bien connus tels que ceux de Lagrange, de Poisson, de Kirchioff, de M. Boussinesq, de M. Maurice LEvy, etc. Non seulement les méthodes employées par ces différents auteurs sont très diverses, mais les résultats eux-mêmes n'ont pas toujours été concordants.

Il semble d'ailleurs que les méthodes auxquelles nous venons de faire allusion puissent, tout au moins lorsqu'on admet, comme on doit le faire, l'existence de pressions extérieures exercées sur les faces de la plaque, être aujourd'hui améliorées dans le sens de la rigueur mathématique; autrement dit dans le sens indiqué par M. Hilbert dans une communication récente. $\dagger$ C'est ce que j'ai tenté de faire dans ce qui va suivre. Je n'y ai d'ailleurs que fort incomplètement réussi ; cet insuccès tient peut-être, nous le verrons, à ce que les problèmes physiques les plus simples peuvent être en relation avec des questions mathématiques difficiles et non encore abordées.

I.

Le problème qui nous occupe est, en effet, au moins théoriquement, l'un des plus simples et de ceux dont la mise en équation semble devoir offrir le moins de difficultés. Il n'est autre chose qu'un cas particulier d'un problème connu, puisqu'une plaque n'est qu'un corps solide de forme particulière.

Cette circonstance doit nous faire abandonner toute une catégorie de méthodes qui ont été quelquefois proposées pour arriver à notre objet actuel. Je veux parler de celles qui consistent à traiter la question directement, sans faire intervenir les équations générales de l'élasticité, en partant d'hypothèses plus ou moins analogues à celles qui ont servi à établir ces équations. Il est clair que de telles hypothèses sont nécessairement des conséquences des premières à moins qu'elles ne soient en contradiction avec elles, et que, dans les deux cas, elles doivent être rejetées. Nous aurons, au contraire, à utiliser les équations générales de l'élas-

* Presented to the Society at the Ithaca meeting, October 26, 1901. Received for publication March 31, 1902.

† Congrès des Mathématiciens, Paris, 1900. 
ticité en faisant des hypothèses spéciales - hypothèses toujours vérifiées dans les conditions où le phénomène se présente expérimentalement * - sur les données du problème : autrement dit, sur la forme du corps étudié (celui-ci devant avoir une dimension très petite par rapport aux deux autres et être, en tous ses points, très voisin du plan des $x y$ ) et sur la nature des forces qui lui sont appliquées.

Les hypothèses que nous ferons à ce dernier point de vue consisteront a èxclure certaines distributions de forces exceptionelles, autrement dit, présentant certaines relations particulières d'égalité approchée (l'ordre d'approximation étant celui de l'épaisseur $2 \epsilon$ de la plaque).

Ces hypothèses seront enoncées en leur lieu. Mais nous serons obligés de leur en adjoindre une autre qui, bien qu'ayant été adoptée comme évidente par tous les auteurs qui ont traité de ces questions, ne se justifie pas par les mêmes raisons que celles dont nous venons de parler, et par conséquent, devra être rejetée dans une théorie définitive.

Cette hypothèse concerne la différentiation des quantités qui vont intervenir dans les calculs. Les quantités en question dépendent des trois coordonnées $x$, $y, z$ dont les deux premières, ainsi que nous l'avons dit, déterminent la projection du point sur le plan horizontal (le plan de la plaque) pendant que la troisième correspond au déplacement en épaisseur. Supposons qu'une des quantités etudiées (déplacements, tensions, etc.) soit, dans toute l'étendue de la plaque, d'un certain ordre de petitesse. Nous admettrons que cet ordre est aussi celui des quantités obtenues en différentiant la première par rapport à $x$ ou à $y$.

Il est clair qu'un tel fait n'est nullement évident à priori. Si la quantité $U$ est, en valeur absolue, plus petite qu'un certain nombre $P$, en tout point de la plaque, la formule des accroissements finis montre bien que sur tout segment parallèle a l'axe des $x$ et dont la longueur n'est pas très petite, il existe des points dans lesquels $\partial U / \partial x$ est inférieur, en valeur absolue, à une quantité assignable et dans un rapport fini avec $P$. Mais rien ne prouve que ces points ne soient pas séparés par d'autres en lesquels cette relation de grandeur cesse d'avoir lieu. C'est ce qui arriverait si la quantité $U$ était soumise à des oscillations très rapides; par exemple, si $U$ était de la forme

$$
U=A \sin \frac{x}{\epsilon} .
$$

Ce fait, qui n'est d'ailleurs point relatif aux données de la question, mais aux propriétés de la solution cherchée, devrait donc, en tout rigueur, être démontré. Nous l'admettrons néanmoins dans ce qui va suivre.

Par contre, la même hypothèse ne sera pas faite en ce qui concerne la différentiation par rapport à $z$. Nous ne pouvons pas, en effet, refaire pour cette

\footnotetext{
* C'est ainsi qu'il n'est pas légitime de supposer nulles les pressions exercées sur les faces de a plaque.
} 
dernière le raisonnement qui vient de nous servir pour les dérivations relatives a $x$ ou à $y$. Une quantité étant de l'ordre de $P$ dans toute notre plaque, il se pourrait fort bien que sa dérivée, par rapport a $z$ fut partout de l'ordre de $P / \epsilon$.

De même, nous n'admettrons l'hypothèse précédente que dans les parties de la plaque suffisament éloignées des bords. Pour les points dont la distance à ceux-ci est de l'ordre de $\epsilon$, nous ne la considérerons plus comme légitime.

D'après ce qui précède, nous admettrons que les dérivées des projections $u$, $v, w$, du déplacement sont (sauf près des bords) du même ordre de grandeur que ces projections elles-mêmes. Ce fait est, au reste, considéré comme évident dans tous les travaux, qui traitent de l'élasticité: nous ne l'accepterons pas ici pour les dérivées par rapport à $z$.

II.

Les équations que nous écrirons ne sont autres, au fond, que celles qui interviennent dans l'exposition donnée de cette théorie par de SaInt Venant.* Elles n'en diffèrent que par une évaluation plus rigoureuse des ordres de grandeur. Nous nous conformerons done aux notations de l'ouvrage en question, et nous désignerons par

$$
\mathfrak{g}_{x}=\frac{\partial u}{\partial x}, \mathfrak{g}_{y}=\frac{\partial v}{\partial y}, \ldots ; \quad \mathrm{g}_{y s}=\frac{\partial v}{\partial z}+\frac{\partial w}{\partial y}, \ldots, \ldots,
$$

les composantes de la déformation : par $t_{x x}, t_{x y}, \ldots$ celles de l'effort ; par $A, B$, $C$, les composantes de la force agissante par unité de masse ; par $2 \epsilon$ l'épaisseur de la plaque. Les coefficients d'élasticité seront supposés tous du même ordre de grandeur. $\dagger$ Soit $k$ une quantité de cet ordre de grandeur.

Soit $k P$ le maximum des tensions $t_{x x}, t_{x y}$, etc. En vertu de notre hypothèse de différentiation, les derivées $\partial t_{x x} / \partial x, \cdots, \partial t_{y y} / \partial y$ seront, au plus (en tout point suffisament éloigné du bord) de l'ordre de grandeur de $k P$. Pour abréger l'écriture, nous désignerons encore par $P$ toute quantité dont le rapport à $P$ n'est pas très grand. Dans ce système de notation, par conséquent, on pourra écrire

$$
\frac{\partial t_{x x}}{\partial x}=k P, \quad \frac{\partial t_{y y}}{\partial y}=k P, \quad \cdots
$$

* Clebsch, Théorie de l'élasticité des corps solides. Traduction de Saint Venant et Flamant, ch. 5 , pages $689-733$ (Note du $\$ 73$ ) ; 1883.

† Plus exactement, soit $k_{1}$ le plus grand des coefficients d'élasticité, c'est à dire des coeffioients par lesquels il faut multiplier les composantes de la déformation pour obtenir les tensions $t_{x x}, \cdots$. Inversement, exprimons les composantes $\mathfrak{g}_{x}, \cdots$ en fonction des tensions : soit $l_{2}$ le plus grand coefficient qui figure dans les équations linéaires ainsi obtenues. Nous supposerons que le produit $k_{1} l_{2}$ (qui est un pur nombre) n'est pas un nombre très grand; qu'en particulier, le produit $k_{1} l_{2} \varepsilon$ est une des quantités que nous regardons comme du même ordre de grandeur que $\varepsilon$. 
Prenons alors la première équation de l'équilibre interne

$$
\frac{\partial t_{x x}}{\partial x}+\frac{\partial t_{x y}}{\partial y}+\frac{\partial t_{x z}}{\partial z}+A=0 .
$$

Multiplions la par $d z$ et, ainsi que le fait de Saint Venant, intégrons de $-\epsilon$ à $z$ (où $z$ est compris entre $-\epsilon$ et $+\epsilon$ ). Le résultat est manifestement, d'après les relations (1), de la forme

$$
t_{x z}-\left(t_{x z}\right)_{-\mathrm{e}}+\int_{-\mathrm{e}}^{z} A d z=k \epsilon P .
$$

En particulier, pour $z=+\epsilon$, il vient

$$
\left(t_{x z}\right)_{+\mathrm{e}}-\left(t_{x z}\right)_{-\mathrm{e}}+\int_{-\mathrm{e}}^{+\mathrm{e}} A d z=k \epsilon P .
$$

Or, les valeurs des tensions $t_{x z}$ sont supposées données sur les deux faces de la plaque. Si ces quantités, ainsi que l'expression

$$
\int_{-e}^{+e} A d z
$$

n'étaient pas toutes de l'ordre de $k \epsilon P$, il faudrait qu'il y eût, entre les pressions exercées sur les deux faces et les forces agissant sur la masse, des relations particulières comme il s'en produirait, par exemple, si l'on agissait à l'endroit considéré sur les deux faces avec les deux branches d'un étau ; ou encore si, la plaque étant soumise à son propre poids, on exerçait sur elle une pression qui neutralise à peu près exactement ce poids. Ce sont précisément de telles relations que nous nous sommes réservé, en commençant, le droit d'exclure. Nous devons donc admettre que dans la somme

$$
\left(t_{x z}\right)_{+\epsilon}-\left(t_{x z}\right)_{-\epsilon}-\int_{-\epsilon}^{+e} A d z
$$

chacun des termes est (abstraction faite des bords dont nous parlerons plus loin) au plus du même ordre de grandeur que la plus grande valeur prise par la somme entière, autrement dit, que $t_{x z}$ est, sur les deux faces et, par conséquent (en vertu de la relation (2)) dans toute la masse, de l'ordre de $k \in P$. De plus il en est de même de l'intégrale

$$
\int_{-\mathrm{e}}^{+\epsilon} A d z
$$

et comme la force $A$ est, dans la pratique, toujours sensiblement constante, on doit admettre que cette force est dans un rapport fini avec $k P$.

Pareillement, $t_{y z}$ est de la forme $k \in P$ et $B$ de l'ordre de $k P$. 
Prenons maintenant la troisième équation de l'équilibre interne

$$
\frac{\partial t_{x z}}{\partial x}+\frac{\partial t_{y z}}{\partial y}+\frac{\partial t_{z z}}{\partial z}+C=0 .
$$

Si nous admettons, conformément a notre hypothèse générale, que $\partial t_{x z} / \partial x, \partial t_{y z} / \partial y$ sont du même ordre que $t_{x z}, t_{y z}$, cette équation, intégrée par rapport à $z$, nous donnera, comme tout à l'heure,

$$
\left(t_{z z}\right)_{+\epsilon}-\left(t_{z z}\right)_{-\epsilon}+\int_{-\epsilon}^{+\epsilon} C d z=k \epsilon^{2} P .
$$

Ici encore, à moins de données exceptionelles pour lesquelles, en effet, les conclusions se trouveraient modifiées, nous avons le droit, d'après cette équation, de faire l'hypothèse que $t_{z z}$ est partout du même ordre que $k \epsilon^{2} P$.

Ainsi les tensions $t_{x z}, t_{y z}$ sont très petites par rapport à $t_{x x}, t_{x y}, t_{z y}$, et la tension $t_{z z}$ est très petite par rapport aux premières. On voit que cette conclusion, que de Saint Venant justifie en préjugeant à priori de l'allure des phénomènes,* est démontrable en toute rigueur, du moins une fois admise notre hypothèse de différentiation. Toutefois, les conclusions auxquelles nous venons de parvenir comportent une restriction essentielle: elles n'ont plus lieu dans le voisinage du bord de la plaque. Cela tient, d'une part à ce que nous abandonnons dans cette région, l'hypothèse de différentiation, utilisée par nous dans le raisonnement qui précède; d'autre part, à ce que les hypothèses faites tout à l'heure sur la distribution des pressions données sur les faces ne sont plus, ici, vérifiées dans les conditions ordinaires des expériences. Ce sont, en effet, des actions combinées (telles que celles des deux branches d'un étau) qu'on exerce souvent sur les contours des plaques, de sorte que la concordance particulière dont nous avons exclu la possibilité dans les parties médianes se présente, au contraire, aux bords. Nous n'admettrons donc nullement, dans le voisinage de ceux-ci, que $t_{z x}, t_{z y}, t_{z z}$ soient de la forme $k \epsilon P$ ou $k \epsilon^{2} P$.

Quant à la quantité $C$, elle devra être au plus de l'ordre de $k \in P$. Au reste,

* Par exemple, l'auteur, après avoir indiqué (loc. cit., p. 697) les intégrations par rapport à $z$ que nous lui empruntons dans le texte, ajoute "on voit que les composantes $t_{z x}$ aux $t_{z y}$ n'ont de valeurs, à l'intérieur d'un tronçon de plaque, que celles qu'elles peuvent avoir sur une des deux bases, plus ce qui vient des forces $A, B$ agissant sur sa masse. Ces forces locales n'ont qu'une influence insignifiante qui n'est presque rien en comparaison de ce qui vient à la fois de toutes les forces agissant sur le reste de la plaque ainsi que sur ses bords, par les réactions des appuis ou autrement, et dont les effets accumulés se transmettent au tronçon à travers ses quatre faces latérales, ce qui s'applique surtout aux composantes agissant horizontalement."

$\mathrm{Au}$ reste, le défaut d'une évaluation rigoureuse pour les ordres de grandeur se fait encore sentir dès le troisième alinéa de la page 697, dont il parait malaisé de préciser le sens. Il semblerait, en effet, résulter de cet alinéa que les derivées des tensions $t_{x x}, t_{x y}, t_{y y}$ par rapport à $x$ et à $y$ sont négligeables devant ces tensions elles-mêmes: assertion manifestement inexacte, qui n'a pu entrer dans la pensée de l'auteur. 
dans la plupart des cas usuels, les forces $A, B, C$ sont de beaucoup inférieures aux limites mêmes que nous venons de leur assigner et n'ont qu'une influence négligeable.

III.

Les coefficients d'élasticité étant supposés d'un même ordre de grandeur $k$, les équations

$$
\begin{gathered}
t_{x x}, \quad t_{x y}, \quad t_{y y}=k P, \\
t_{x x}, t_{y z}=k \epsilon P ; \quad t_{z x}=k \epsilon^{2} P,
\end{gathered}
$$

montrent évidemment que les six composantes de déformation sont de l'ordre de $P$. De plus, si l'on résout les trois dernières d'entre elles par rapport à $\mathrm{g}_{z x}, \mathrm{~g}_{x y}, \mathfrak{g}_{z}$ en y remplaçant les seconds membres par zéro, on ne fera sur les valeurs de ces inconnues que des erreurs de l'ordre de $\epsilon P$.

Or les équations que l'on obtiendrait ainsi sont

$$
\frac{\partial W}{\partial \mathrm{g}_{z x}}=0, \quad \frac{\partial W}{\partial \mathrm{g}_{z y}}=0, \quad \frac{\partial W}{\partial \mathfrak{g}_{z}}=0 .
$$

$W$ étant l'énergie interne spécifique.

Soit $W_{1}\left(\mathfrak{g}_{x}, \mathrm{~g}_{x y}, \mathfrak{g}_{y}\right)$, ce que devient $W$ lorsqu'on y met pour $\mathrm{g}_{z x}, \mathrm{~g}_{x y}, \mathfrak{g}_{x}$ leurs valeurs tirées des équations (5). On a, moyennant les mêmes équations,

$$
\frac{\partial W}{\partial \mathfrak{g}_{x}}=\frac{\partial W_{1}}{\partial \mathfrak{g}_{x}}, \frac{\partial W}{\partial \mathrm{g}_{x y}}=\frac{\partial W_{1}}{\partial \mathrm{g}_{x y}}, \frac{\partial W}{\partial \mathfrak{g}_{y}}=\frac{\partial W_{1}}{\partial \mathfrak{g}_{y}} .
$$

Dès lors (puisque nous ferions sur $\mathrm{g}_{x z}, \mathrm{~g}_{y z}, \mathfrak{g}_{z}$, en écrivant les équations (5), des erreurs de l'ordre de $\epsilon P$ ), les expressions de $t_{x x}, t_{x y}, t_{y y}$ seront de la forme

$$
t_{x x}=\frac{\partial W_{1}}{\partial \mathfrak{g}_{x}}+k \epsilon P, \quad t_{x y}=\frac{\partial W_{1}}{\partial \mathrm{g}_{x y}}+k \epsilon P, \quad t_{y y}=\frac{\partial W_{1}}{\partial \mathfrak{G}_{y}}+k \epsilon P .
$$

Considérons maintenant la dérivée $\partial^{2} w / \partial z \partial x$. En vertu de notre hypothèse de différentiation, cette quantité sera du même ordre que $\partial w / \partial z$. c'est à dire d'ordre $P$.

Soient alors $u_{0}, v_{0}, w_{0}$ les valeurs de $u, v, w$ au feuillet moyen (l'ordonnée z étant comptée à partir de ce feuillet). On aura

Mais l'équation

$$
\frac{\partial w}{\partial x}=\frac{\partial w_{0}}{\partial x}+\epsilon P
$$

$$
\mathrm{g}_{x x}=\frac{\partial z}{\partial u}+\frac{\partial w}{\partial x}=P
$$

donnera, dans ces conditions 


$$
u=u_{0}-z \frac{\partial w_{0}}{\partial x}+\epsilon P
$$

et l'on aura, de même

$$
v=v_{0}-z \frac{\partial w_{0}}{\partial y}+\epsilon P
$$

d'où

$$
\begin{aligned}
& t_{x x}=t_{x x}^{0}-z t_{x x}^{\prime}+k \epsilon P, \\
& t_{x y}=t_{x y}^{0}-z t_{x y}^{\prime}+k \epsilon P, \\
& t_{y y}=t_{y y}^{0}-z t_{y y}^{\prime}+k \epsilon P,
\end{aligned}
$$

$t_{x x}^{\prime}, t_{x y}^{\prime}, t_{y y}^{\prime}$ étant ce que deviennent $t_{x x}, t_{x y}, t_{y y}$ en vertu des équations (6) et au terme $k \epsilon P$ près quand on $\mathrm{y}$ remplace $u$ par $\partial w_{0} / \partial x$ et $v$ par $\partial w_{0} / \partial y$, soit, symboliquement

$$
\begin{aligned}
& t_{x x}^{\prime}=\frac{\partial W_{1}}{\partial \mathfrak{G}_{x}}\left(\frac{\partial^{2}}{\partial x^{2}}, \quad 2 \frac{\partial^{2}}{\partial x \partial y}, \frac{\partial^{2}}{\partial y^{2}}\right) w_{0}, \\
& t_{x y}^{\prime}=\frac{\partial W_{1}}{\partial \mathrm{g}_{x y}}\left(\begin{array}{ccc}
\frac{\partial^{2}}{\partial x^{2}}, & 2 \frac{\partial^{2}}{\partial x \partial y}, & \frac{\partial^{2}}{\partial y^{2}}
\end{array}\right) w_{0},
\end{aligned}
$$

Dès lors le calcul fait au no. 3 (pages 694 à 696 ) de la Note citée de SaINT Venant conduit à l'équation

$$
2 \frac{\epsilon^{3}}{3} W_{1}\left(\begin{array}{ccc}
\frac{\partial^{2}}{\partial x^{2}}, & 2 \frac{\partial^{2}}{\partial x \partial y}, & \partial^{2} \\
\partial y^{2}
\end{array}\right) w_{0}=f(x, y)+k \epsilon^{3} P,
$$

où $f(x, y)$ a l'expression donnée par de Saint Venant (no. 3, équation $(f)$ ). De même, on a tous les éléments nécessaires pour effectuer le calcul présenté au no. 7 de la même Note ; et l'on aura des équations tout analogues à celles obtenues en cet endroit, (équations $(z),\left(z^{\prime}\right)$ ), mais qui devront être complétées par des termes de la forme $k \epsilon^{2} P$.

$$
\text { IV. }
$$

Avant de chercher à évaluer la quantité $P$, nous nous occuperons de former les conditions aux limites, question qui a été, comme on le sait, la source des plus grandes difficultés soulevées par la théorie des plaques. La solution de ces difficultés doit, comme on le sait également, être cherchée dans l'état particulier des bords de la plaque, lesquels éprouvent des déformations autres que les régions intérieures. Un tel fait s'introduit tout naturellement d'après ce qui précède, puisque nous renonçons à admettre, pour ces bords, les hypothèses qui ont servi de base à nos raisonnements précédents.

Nous tracerons done, à une petite distance (de l'ordre de $\epsilon$ ) du bord de la plaque, ${ }^{*}$ un cylindre vertical $C$. A l'intérieur de ce cylindre, les conclusions

* Les conclusions que nous allons obtenir subsistent dans ce qu'ils ont d'essentiel, si la distance en question est moindre que $\varepsilon^{1-a}, a$ étant un nombre positif suffisament petit. 
précédemment obtenues seront valables. Au contraire, dans la région annulaire (que nous appelerons la marge) extérieure à $C$ nous ne saurons plus qu'une chose, c'est que les diverses tensions $t_{x x}, t_{x y}, t_{y y}, t_{x z}, t_{y z}, t_{z z}$, sont au plus, de l'ordre de $k P$.

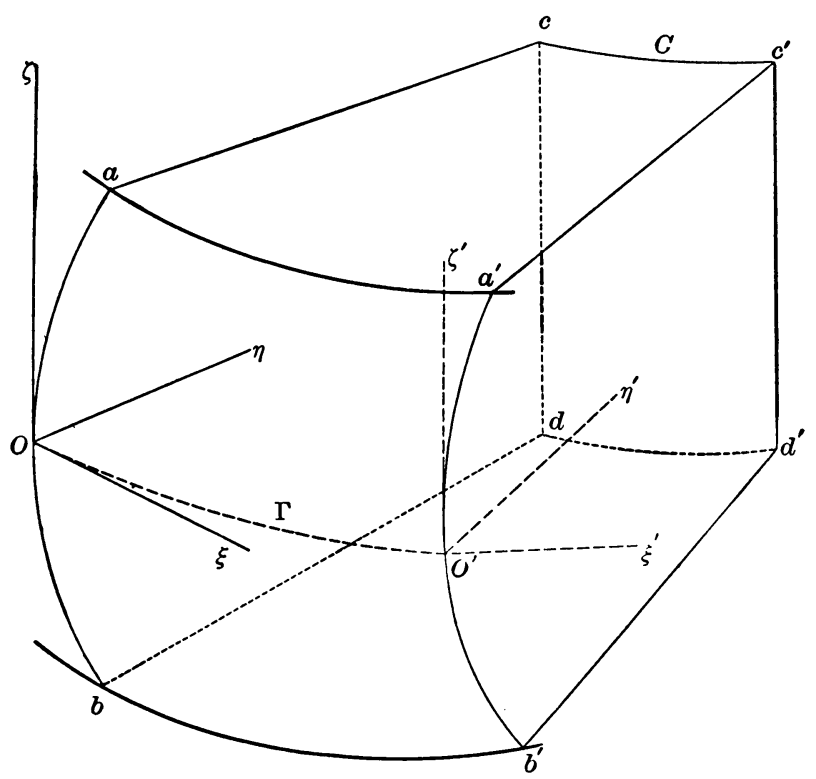

Fig. 1.

Menons, normalement au contour $\Gamma$ de la plaque en un point quelconque de celui-ci, un plan qui coupera le cylindre $C$ suivant une génératrice $c d$ et la marge suivant le polygone mixtiligne $a b c d$ (voir la fig. 1). Soient $O$ un point de la section ainsi obtenue (par exemple comme sur la figure, un point situé sur le contour); $O \xi, O \eta, O \zeta$, trois axes, dírigés l'un suivant la tangente au contour $\Gamma$, le second, suivant la normale horizontale à cette courbe, le troisième, suivant la verticale. L'ensemble des forces élastiques qui s'exercent sur un des cotés de la section abcd aura, par rapport aux axes $O \xi, O \eta, O \zeta$, trois projections que nous désignerons par $X, Y, Z$, trois moments désignés par $\mu_{\bullet}, \mu_{n}, \mu_{\varepsilon}$, les ordres de grandeur de ces diverses quantités étant évidemment au plus

$$
X, Y, Z=k \epsilon^{2} P, \quad \mu_{s}, \mu_{n}, \mu_{z}=k \epsilon^{3} P .
$$

Ainsi qu'il a été dit plus haut, l'hypothèse relative à la différentiation est conservée pour les différentiations le long du contour. Donc les quantités $d X / d s$ ( $d s$ étant l'élément d'are de $\Gamma$ ) $, d Y / d s, d Z / d s$ sont encore de l'ordre de $k \epsilon^{2} P$; les quantités $d \mu_{s} / d s, d \mu_{n} / d s, d \mu_{z} / d s$, de l'ordre de $k \epsilon^{3} P$.

La section $a b c d$ et une section voisine $a^{\prime} b^{\prime} c^{\prime} d^{\prime}$, séparée de la première par un arc $d s \mathrm{du}$ contour, intercepteront entre elles une portion de la tranche verticale 
de la plaque, et l'ensemble des pressions extérieures exercées sur cette petite surface aura, par rapport aux axes $O \xi, O \eta, O \zeta$, trois moments que nous désignerons, conformément aux notations de l'ouvrage cité, par $M_{s} d s, M_{n} d s, 0:{ }^{*}$ et trois projections que nous désignerons par $S^{\prime} d s, N^{\prime} d s, W^{\prime} d s$.

Ecrivons l'équilibre du solide $a b c d a^{\prime} b^{\prime} c^{\prime} d^{\prime}$. Celui-ci est soumis :

$1^{\circ}$. Aux forces extérieures données.

$2^{\circ}$. Aux forces élastiques développées sur la face $a b c d$.

$3^{\circ}$. Aux forces élastiques développées sur la face $a^{\prime} b^{\prime} c^{\prime} d^{\prime}$. Si, par rapport à cette face, on mène les axes $O^{\prime} \xi^{\prime}, O^{\prime} \eta^{\prime}, O^{\prime} \zeta^{\prime}$ analogues à $O \xi, O \eta, O \zeta$, les forces élastiques en question auront pour projections et pour moments par rapport à ces axes $X+d X, Y+d Y, Z+d Z, \mu_{s}+d \mu_{s}, \mu_{n}+d \mu_{n}, \mu_{z}+d \mu_{z}$. On en déduit, par un calcul élémentaire, en tenant compte de l'angle $d \theta=d s / \rho$ que fait $O^{\prime} \xi^{\prime}$ avec $O \xi$, les projections et les moments de ces mêmes forces par rapport aux axes $O \xi, O \eta, O \zeta$.

$4^{\circ}$. Les forces élastiques qui s'exercent suivant la face $c d c^{\prime} d^{\prime}$. Ces dernières s'évaluent à l'aide des formules précédemment écrites, comme il est expliqué dans la note citée de Saint Venant (noṣ. 13-18).

L'équation des moments par rapport à $O \zeta$ ne nous fournit aucun renseignement, car, les quantités qui y figurent, et dont les unes sont à conserver dans le résultat, les autres à éliminer, sont toutes du même ordre de grandeur que $k \epsilon^{2} P$.

Les équations des projections horizontales nous donnent les formules $\left(o_{1}\right)$, $\left(o_{0}^{\prime}\right)$ (no. 14) de Saint Venant complétées par des termes de la forme $k \epsilon^{2} P$ soit, lorsque la plaque est isotrope,

$$
k \epsilon^{2} P+2 \epsilon\left\{\left[\mathrm{a}_{1}\left(\frac{\partial u_{0}}{\partial x}+\frac{\partial v_{0}}{\partial y}\right)-2 \mathrm{f} \frac{\partial \dot{\dot{v}}}{\partial y}\right] \cos p+\mathrm{f}\left(\frac{\partial u_{0}}{\partial y}+\frac{\partial v_{0}}{\partial x}\right) \sin p\right\}
$$

$$
\begin{aligned}
k \epsilon^{2} P+2 \epsilon\left\{\left[\mathrm{a}_{1}\left(\frac{\partial u_{0}}{\partial x}+\frac{\partial v_{0}}{\partial y}\right)-2 \mathrm{f} \frac{\partial u_{0}}{\partial x}\right] \sin p\right. & \left.+\mathrm{f}\left(\frac{\partial u_{0}}{\partial y}+\frac{\partial v_{0}}{\partial x}\right) \cos p\right\} \\
& =V^{\prime}=S^{\prime} \cos p+N^{\prime} \sin p
\end{aligned}
$$

$p$ étant l'angle de la normale $O \eta$ avec l'axe des $x$.

Dans l'équation des moments de flexion, s'introduisent les moments $\mu_{s}$ et $\mu_{n}$ (ainsi que leurs derivées par rapport à $s$ ). Ces moments sont de la forme $k \epsilon^{3} P$ et ne font que se joindre aux termes du même ordre provenant des seconds membres des équations (8), de sorte qu'il vient (comparer Saint Venant, équation $\left(t_{1}\right)$, no. 16, p. 713$)$,

$$
-\frac{2 \epsilon^{3}}{3}\left(t_{x x}^{\prime} \cos ^{2} p+2 t_{x y}^{\prime} \sin p \cos p+t_{y y}^{\prime} \sin ^{2} p\right)=M_{s}+k \epsilon^{3} P .
$$

\footnotetext{
* On sait que le moment par rapport à $O \zeta$ est de l'ordre de $d s^{2}$ et non de $d s$.
} 
Prenons, au contraire, les équations des projections verticales et des moments de torsion : nous aurons

$$
W^{\prime}=\frac{\partial Z}{\partial s}-\frac{2 \epsilon^{3}}{3}\left[\cos p\left(\frac{\partial t_{x x}^{\prime}}{\partial x}+\frac{\partial t_{x y}^{\prime}}{\partial y}\right)+\sin p\left(\frac{\partial t_{x y}^{\prime}}{\partial x}+\frac{\partial t_{y y}^{\prime}}{\partial y}\right)\right]+Q+k \epsilon^{3} P
$$

$$
\begin{aligned}
M_{n}=-Z+\frac{d \mu_{n}}{d s}-\frac{\mu_{s}}{\rho}+\frac{2 \epsilon^{3}}{3} \frac{\partial}{\partial s}[\sin p \cos p & \left(t_{x x}^{\prime}-t_{y y}^{\prime}\right) \\
& \left.+\left(\cos ^{2} p-\sin ^{2} p\right) t_{x y}^{\prime}\right]+k \epsilon^{3} P
\end{aligned}
$$

$\mathbf{Q}$ étant une quantité connue, fonction des efforts donnés, et qui figure dans l'équation $\left(d_{2}\right)$ (page 716) de SaINT Venant.

Les moments $\mu_{\varepsilon}$ et $\mu_{n}^{\prime}$ sont, nous l'avons dit, de l'ordre de $k \epsilon^{3} P$. Mais il n'est pas de même de $Z$. Si donc nous ne voulons conserver (outre les termes qui nous intéressent) que des quantités de l'ordre de $k \epsilon^{3} P$, il faut s'éliminer $Z$ entre les deux équations précédentes, c'est à dire former la combinaison de KIRCHнOFF.

Il y a lieu d'observer que l'existence d'efforts verticaux considérables exercés sur les bords de la plaque ne suffirait pas, à elle seule, à introduire notre quantité $Z$. S'il en était ainsi, en effet, $Z$ serait encore négligeable (à savoir de l'ordre de $k \epsilon^{3} P$ ), en tous les points du contour où les efforts verticaux donnés seraient nuls ou du même ordre que dans l'intérieur. Or cela n'a pas lieu: c'est ce dont il est facile de s'assurer dans des cas particuliers (tels que celui de la plaque circulaire) et c'est ce qui résulte à priori de ce que pour $Z$ négligeable, on aurait à écrire séparément les deux équations (14), ce qui donnerait trois conditions aux limites, au lieu de deux, sur certaines parties du contour.

Il est donc nécessaire, non seulement d'admettre éventuellement l'existence d'efforts autres que ceux qui peuvent avoir lieu dans l'intérieur, mais encore d'abandonner, près des bords, l'hypothèse de différentiation.

V.

Pour évaluer l'ordre de grandeur de $P$, on peut partir d'une hypothèse classique dans cette théorie.

On sait qu'un solide dont une dimension est très petite peut être déformé de manière que les composantes de la déformation en chaque point soient très petites par rapport aux déplacements (même aux déplacements relatifs) de ces points, le rapport de ces composantes à ces déplacements étant de l'ordre de la dimension très petite.

Convenons d'appeler flexion toute déformation qui satisfait à la condition précédente.

L'hypothèse dont.il s'agit consiste à admettre que la déformation cherchée est une flexion. 
Dans ce cas, la quantité que nous avons designée par $P$ sera très petite par rapport au plus grand des déplacements $u, v, w$ cherchés : elle sera comparable au produit de ce déplacement par $\epsilon$.

Dès lors, la question peut être considérée commé résolue. Car la quantité $k \epsilon^{3} P$ pourra être négligée, comme étant d'ordre supérieur au premier membre, dans l'équation (10). De même, les quantités de la forme $k \epsilon^{3} P$ par lesquelles nous avions complété les équations $(z),\left(z^{\prime}\right)$ pourront être négligées, et ces équations regardées comme exactes.

De plus, si nous supposons que les tensions conservent encore le même ordre de grandeur dans le voisinage des bords, nous pourrons faire abstraction des termes $k \epsilon^{2} P$ des équations (12) et $k \epsilon^{3} P$ des équations (13), (14).

L'hypothèse ainsi utilisée est bien, contrairement à ce qui parait au premier abord, une hypothèse sur les données du problème et que nous avons le droit de faire.

Supposons, en effet, les efforts $F$ qui agissent sur la plaque decomposés en deux parties $F^{\prime}, F^{\prime \prime}$, dont la première, agissant seule, produirait une flexion, la seconde non. Supposons de plus que les efforts $F^{\prime}$ ne soient pas très petits par rapport aux $F^{\prime \prime}$. Alors les composantes de déformation produites en chaque point par $F^{\prime}$ ne seront pas très petites par rapport à celles qui sont dues à $F^{\prime \prime}$. Or ceci exige, d'après ce que nous supposons sur $F^{\prime}$ et $F^{\prime \prime}$, que les déplacements produits par $F^{\prime}$ soient très grands par rapport à ceux qui correspondent à $F^{\prime \prime}$. La déformation sensible à l'oeil sera donc une flexion, sauf dans le cas tout exceptionnel où les efforts capables de produire une flexion seraient infiniment petits par rapport aux autres.

Dans ce cas, les résultats pourraient être fort différents : la déformation produite serait évidemment beaucoup plus petite pour la même intensité d'effort que dans les circonstances habituelles.

Le fait que la déformation obtenue est une flexion est donc bien une hypothèse sur les données du problème, puisqu'il peut avoir lieu, ou non, suivant la nature de ces données; et c'est bien une hypothèse légitime puisqu'elle n'est en défaut que dans des cas exceptionnels (et qu'il serait même assez difficile de réaliser expérimentalement).

Toutefois cette manière d'opérer soulève quelques critiques. D'abord, le raisonnement précédent n'est valable que si l'on est certain qu'il existe des flexions, c'est à dire que leur existence est compatible non seulement (comme il est bien connu) avec les données de la géometrie, mais encore avec celles de la Mécanique, avec les équations de l'équilibre élastique.

Ce point résulte (du moins en l'absence de forces agissant sur les éléments de masse) des résultats de M. MaUrice Levr.* Celui-ci a écrit les équations d'une infinité de déformations satisfaisant aux équations de l'élasticité et qui

\footnotetext{
* Journal de Mathématiques, 3 Série, t. 3, 1877.
} 
sont des flexions. On peut d'ailleurs établir directement l'existence de déformations de cette espèce en partant des flexions dont l'existence a été établie au point de vue géométrique. Il suffira de calculer les forces $X, Y, Z$ qu'il est nécessaire de faire agir sur l'unité de masse du corps pour le maintenir en équilibre dans ces conditions. Ces quantités sont de l'ordre des forces élastiques correspondant aux déformations considérées, c'est à dire du produit de $k \epsilon$ par le déplacement. Si alors nous considérons un corps solide de dimensions finies en tous sens et dont fasse partie la plaque donnée, et que nous fassions agir sur ses différents points des forces coïncidant avec $X, Y, Z$ (ou, plus généralement, avec $X+A, Y+B, Z+C)$ dans l'intérieur de notre plaque et qui soient du même ordre partout ailleurs, il est clair que les déplacements et les déformations produites dans ces nouvelles conditions seront du même ordre que les composantes de déformation primitives et, par conséquent, très petits par rapport aux déplacements primitifs. En les combinant avec ceux-ci on aura une déformation possédant la propriété cherchée.

Mais on peut faire à la méthode précédente un autre reproche. D'après ce que nous avons dit plus haut, sa légitimité résulte de ce que, pour une même intensité de forces agissantes, les déformations qui possèdent le caractère de flexion sont beaucoup plus intenses que les autres. Mais ce fait, lui aussi, est une conséquence nécessaire, mathématique, des données générales de l'élasticité. Il devrait donc pouvoir être mis en évidence par nos calculs. Or, ce n'est point ce qui a lieu dans la théorie que nous venons d'exposer. *

VI.

On peut arriver à un résultat plus satisfaisant si l'on emploie un théorème, non demontré, il est vrai, mais que les progrès accomplis dans ces dernières années permettront sans doute d'établir aisément.

Nous avons vu que $u_{0}, v_{0}$ sont liés aux forces données, d'une part, par deux équations aux derivées partielles analogues aux équations $(z),\left(z^{\prime}\right)$ de SAINT Venant, d'autre part, par les conditions aux limites (12). Soient donc les équations aux derivées partielles $\dagger$

$$
\begin{gathered}
\mathbf{a}_{1} \frac{\partial}{\partial x}\left(\frac{\partial u_{0}}{\partial x}+\frac{\partial v_{0}}{\partial y}\right)-\mathbf{f} \frac{\partial}{\partial y}\left(\frac{\partial u_{0}}{\partial y}-\frac{\partial v_{0}}{\partial x}\right)=\alpha \\
\mathbf{a}_{1} \frac{\partial}{\partial y}\left(\frac{\partial u_{0}}{\partial x}+\frac{\partial v_{0}}{\partial y}\right)-\mathbf{f} \frac{\partial}{\partial x}\left(\frac{\partial u_{0}}{\partial y}-\frac{\partial v_{0}}{\partial x}\right)=\beta,
\end{gathered}
$$

\footnotetext{
* Au reste, cette seule circonstance qu'il suffise de montrer l'existence des flexions, sans qu'il soit besoin de rien prouver sur le degré de généralité de ces sortes de déformations, met en évidence le caractère artificiel de cette manière d'opérer.

† Nous prenons, pour simplifier, les équations relatives à la plaque isotrope.
} 
dans lesquelles les fonctions inconnues $u_{0}, v_{0}$ doivent satisfaire aux conditions aux limites

$$
\left\{\begin{array}{l}
{\left[\mathrm{a}_{1}\left(\frac{\partial u_{0}}{\partial x}+\frac{\partial v_{0}}{\partial y}\right)-2 \mathrm{f} \frac{\partial v_{0}}{\partial y}\right] \cos p+\mathrm{f}\left(\frac{\partial u_{0}}{\partial y}+\frac{\partial v_{0}}{\partial x}\right) \sin p=\gamma} \\
{\left[\mathrm{a}_{1}\left(\frac{\partial u_{0}}{\partial x}+\frac{\partial v_{0}}{\partial y}\right)-2 \mathrm{f} \frac{\partial u_{0}}{\partial x}\right] \sin p+\mathrm{f}\left(\frac{\partial u_{0}}{\delta y}+\frac{\partial v_{0}}{\partial x}\right) \cos p=\delta}
\end{array}\right.
$$

Supposons qu'on ait établi :

$1^{\circ}$. que le problème ainsi posé a une solution, et une seule;

$2^{\circ}$. que les valeurs de $u_{0}, v_{0}$ et de leurs dérivées en tout point appartenant au domaine considéré sont limitées en fonction de $\alpha, \beta, \gamma, \delta$, et de leurs dérivées.

S'il en est ainsi, les composantes $\mathfrak{G}_{x}, \mathfrak{G}_{y}, \mathrm{~g}_{x y}$ sont limitées en fonction de $\alpha$, $\beta, \gamma, \delta$ (et de leurs derivées) en chaque point de feuillet moyen. En se reportant aux équations ( 7$)\left(7^{\prime}\right)$, on voit immédiatement que partout, ces composantes sont limitées en fonction de $\alpha, \beta, \gamma, \delta, \epsilon w, \epsilon P$. Mais comme les composantes restantes $\mathrm{g}_{x z}, \mathrm{~g}_{y z}, \mathfrak{g}_{z}$ sont liées aux premières par les relations (4), on voit que $P$, qui est comparable à la plus grande valeur des composantes de la déformation, est également limité en fonction de $\alpha, \beta, \gamma, \delta$, et de $\epsilon w$.

Ici, l'on a

$$
\begin{aligned}
& \alpha=k \epsilon P-\frac{1}{2 \epsilon}\left[\left(t_{z x}\right)_{+\epsilon}-\left(t_{z x}\right)_{-\epsilon}+\int_{-\epsilon}^{+\epsilon} A d z\right], \\
& \beta=k \epsilon P-\frac{1}{2 \epsilon}\left[\left(t_{z y}\right)_{+\epsilon}-\left(t_{z y}\right)_{-\epsilon}+\int_{-\epsilon}^{+\epsilon} B d z\right], \\
& \gamma=\frac{U^{\prime}}{2 \epsilon}-k \epsilon P \\
& \delta=\frac{V^{\prime}}{2 \epsilon}-k \epsilon P .
\end{aligned}
$$

Donc $P$ est au plus l'ordre de la plus grande des quantités*

$$
\begin{gathered}
\frac{U^{\prime}}{2 \epsilon}, \frac{V^{\prime}}{2 \epsilon}, \quad \frac{\left(t_{z x}\right)_{+\epsilon}-\left(t_{z x}\right)_{-\epsilon}+\int_{-\epsilon}^{\epsilon} A d z}{2 \epsilon}, \\
\frac{\left(t_{z y}\right)_{+\epsilon}-\left(t_{x y}\right)_{-\epsilon}+\int_{-\epsilon}^{\epsilon} B d z}{2 \epsilon}, \quad \text { et } \epsilon w .
\end{gathered}
$$

* Nous admettons, pour simplifier, que les derivées de ces quantités sont du même ordre qu'elles-mêmes. 
Or, d'autre part, les équations où figure $w$, savoir les équations $(10),(13)$, (14), nous font connaître cette quantité (ou plutôt certaines de ses combinaisons différentielles) par une somme de termes $\boldsymbol{P}$ et de termes dans lesquels les trois quantités connues

$$
f(x, y), \quad M_{s}, \quad W^{\prime}-A+\frac{d M_{n}}{d s},
$$

que l'on peut appeler les efforts verticaux, sont divisées par $\epsilon^{3}$. Donc l'une au moins des inconnues $w, P$ est au moins de l'ordre des quotients ainsi obtenus.

Nous allons faire l'hypothèse, évidemment légitime, que les efforts verticaux ne sont pas très petits par rapport aux efforts horizontaux

$$
U^{\prime}, \quad V^{\prime}, \quad\left(t_{x x}\right)_{+\bullet}-\left(t_{x x}\right)_{-\epsilon}+\int_{-e}^{e} A d z, \quad\left(t_{z y}\right)_{+e}+\left(t_{z y}\right)_{-\epsilon}+\int_{-\epsilon}^{e} B d z .
$$

Alors ce sera certainement $w$ qui sera comparable au quotient des efforts verticaux par $\epsilon^{3}$. Quant a $P$, il sera de l'ordre de $\epsilon w$. Enfin les quantités $\alpha, \beta, \gamma, \delta$ seront de l'ordre de $\epsilon^{2} w$ et il en sera de même des déplacements horizontaux du feuillet moyen, savoir de $u_{0}, v_{0}$.

Au contraire, les déplacements horizontaux des points suffisament éloignés du feuillet moyen seront de l'ordre de $\epsilon w$.

Ces conclusions sont bien celles qu'il s'agissait d'établir et la solution est ainsi achevée.

Ainsi qu'il a été dit plus haut, le lemme de calcul intégral dont je viens de me servir n'a pas été démontré à ma connaissance. Mais les équations aux dérivées partielles (19) ont été étudiées par M. BoGGIO, * avec d'autres conditions aux limites, il est vrai.

Ces conditions correspondent au cas où l'on se donnerait, non les efforts exercés sur les bords, comme nous l'avons fait en général dans ce qui précède, mais les déplacements de ces bords. M. BogGio a, dans ces conditions, établi l'existence de la solution pour des catégories assez étendues de domaines. Il ne restait, on le voit, qu'à étendre ses résultats aux conditions aux limites (16).

\section{VII.}

Nous avons ainsi pu remplacer l'hypothèse consistant à admettre à priori qu'on est en présence d'une flexion par cette autre, irréprochable au point de

* Rendiconti dell'Academia delle Scienze di Torino, 17 décembre 1899 ; N u oro Cimento, série 5, tome I, Mars 1901. Je saisis cette occasion pour indiquer que, dans ne Note inscrée aux Rendiconti dell' Institu to Lombardo (séance du 27 Juin 1901), M. Boggio a traité par des intégrales définies, le problème de l'équilibre de la plaque circulaire libre ou simplement appuyée, que j'ai étudié au même point de vue dans les Annales Scientifiques de l'Ecole Normale supérieure. Mes résultatsavaient été exposée à la Société Mathématique de France dans la séance du 17 avril 1901, mais n'ont été publiés que dans le $\mathbf{n}^{\circ}$. de Septembre de la même année. 
vue des principes adoptés ici, que les efforts horizonitaux ne sont pas très grands par rapport aux efforts verticaux.

Par contre, nous conservons encore dans notre raisonnement deux hypothèses que nous ne sommes pas en état de justifier.

C'est d'abord l'hypothèse de différentiation. Comme nous l'avons vu, celle-ci ne serait pas vérifiée si les diverses quantités que nous considérons présentaient des oscillations à très courtes périodes. Elle introduit donc la notion de ce qu'on pourrait appeler le degré d'oscillation de la quantité en question. C'est une notion évidemment analogue à celle qui intervient souvent, comme l'a fait remarquer M. BoreL,* en théorie des fonctions. L'une est dans le domaine de l'extrêmement petit ce que l'autre est dans le domaine de l'extrêmement grand. Il n'est pas douteux que la notion dont nous venons de parler ne soit fréquemment utile en Physique mathématique et surtout dans les questions d'approximation telles que celle qui nous occupe. La seule difficulté serait de choisir entre les diverses expressions (maximum des dérivées, intégrales portant sur les carrés de ces dérivées, variation au sens de M. Jordan, etc.), susceptibles de mesurer ce degré d'oscillation.

Dans la théorie des fonctions harmoniques, on peut aisément évaluer les degrés d'oscillation. On sait, en effet, que si les valeurs d'une fonction harmonique sont limitées sur la surface d'une sphère, on peut assigner des limites supérieures pour les dérivées de cette fonction en tout point suffisamment ćloigné de la surface. On pourrait chercher à étendre ce résultat aux équations de l'élasticité; mais, dans le problème que nous traitons en ce moment, une difficulté particulière naîtrait évidemment du fait que le domaine envisagé a une dimension extrêmement petite.

On est donc, en somme, ramené $a ̀$ une étude qui donnerait évidemment la solution de tous les problèmes relatifs aux plaques, à savoir celle de l'influence exercée par la forme d'un domaine sur les solutions des équations aux dérivées partielles à caractéristiques imaginaires considérées dans ce domaine. Cette étude reste encore à peu près entièrement à faire. Par exemple, les méthodes qui nous démontrent l'existence des solutions du problème de Dirichlet ne nous renseignent guère sur la manière dont les résultats varient avec la forme du volume donné.

Nous retrouvons d'ailleurs cette même question a un autre point de vue. En disant en effet que la dimension verticale de notre plaque est extrêmement petite, nous entendons évidemment qu'elle est très petite par rapport aux dimensions horizontales. Il faudrait donc faire intervenir celles-ci dans les résultats, autrement dit, savoir comment elles influent sur la limitation des quantités $u_{0}, v_{0}$, solutions des équations. C'est un point sur lequel on est fixé dans une certaine

* Mémoire our les séries divergentes, Annales Scientifiques de l'Ecole Normale Su périe ure, 3 série, t. 16 (1899), ch. 1. 
mesure, mais non d'une manière suffisante, par l'étude de ce qui se passe lorsqu'on remplace le contour donné de la plaque par un contour homothétique.

Remarquons enfin que notre hypothèse de différentiation ne peut manifestement subsister sans restriction, que si elle est vraie pour les efforts donnés, c'est à dire si les derivées de ceux-ci sont du même ordre qu'eux. Malheureusement, ceci cesse d'avoir lieu dans des cas très simples. Il est clair, par exemple, que si l'on pose un corps pesant sur la plaque, l'effort $t_{z z}$ varie très rapidement sur les bords de la région ainsi pressée. Il serait donc nécessaire de modifier les raisonnements pour les étendre aux cas où de pareilles singularités se produiraient.

La seconde hypothèse que nous avons laissé subsister est celle d'après laquelle les efforts dans les parties de la plaque voisine des bords sont du même ordre que dans l'intérieur ou du moins d'après laquelle les résultantes de ces efforts suivant des sections telles que $a b c d$ (Fig. 1) ont l'ordre de grandeur indiqué par les équations (11).

Il n'est pas exact que, dans un corps en équilibre élastique les composantes de l'effort dans le voisinage de la surface soient nécessairement dans un rapport fini avec les valeurs de ces mêmes composantes a l'intérieur. Le contraire aurait lieu, d'après le principe de SaInt Venant, si l'on faisait agir sur une petite région de la surface des forces très grandes se faisant équilibre. Si donc on n'écartait pas ici comme exceptionnel l'existence de telles forces, les conditions aux limites telles que nous les avons obtenues pourraient se trouver modifiées en certains points de contour. Mais il serait sans doute possible de démontrer (une fois établi le principe de SaINT Venant) que ces modifications seraient sans influence sur les valeurs des inconnues dans l'intérieur de la plaque.

D'autre part nous avons fait implicitement une troisième hypothèse qui, toute classique qu'elle soit, il n'en est pas moins nécessaire de rappeler, en réduisant les tensions à leurs termes linéaires par rapport aux composantes de déformation. Rien n'est plus propre que le problème des plaques à montrer combien cette hypothèse mérite d'etre examinée. On sait, en effet, que si la plaque devient une membrane, ou simplement si elle est fortement tendue, les termes du second ordre s'introduisent nécessairement. Au reste, l'influence nécessaire de ces termes du second ordre sur les plaques est mise en évidence par ce fait d'expérience courante que les efforts horizontaux agissent d'une manière toute différente suivant qu'ils sont dilatants ou comprimants. *

\footnotetext{
* Certaines hypothèses sur les données du problème-par conséquent, légitimes au point de vue purement mathématique-mériteraient elles-mêmes d'être reprises. La première de toutes, celle qui consiste à regarder l'épaisseur de la plaque comme très petite relativement aux autres longueurs qui figurent dans la question est du nombre, car des feuilles extrêmement minces devraient être traitées comme des membranes et non comme des plaques, de sorte que des termes du second ordre y deviendraient prépondérants. L'expérience semble montrer qu'il existe des plaques assez épaisses pour que les termes du second ordre par rapport aux déformations puissent
} 
On voit combien de lacunes graves présente encore la théorie précédente, surtout eu égard à la simplicité apparente de la question. Cependant, le lecteur qui prendra la peine de la comparer aux théories antérieurement proposées,-_par exemple, à celle de SaINT Venant, que j'ai suivie dans tout ce qui précède, parce qu'elle est l'étude la plus approfondie qui ait été écrite sur ce sujet-reconnaîtra sans doute qu'elle constitue un progrès notable sur ces dernières.

\section{VIII.}

Lorsque l'on considère une déformation finie de la plaque, l'hypothèse que cette déformation est une flexion doit être forcément adoptée, dans l'état actuel de la question. Si l'on l'abandonnait, en effet, on ne pourrait plus regarder les composantes de déformation comme très petites, et il faudrait introduire la théorie de l'équilibre élastique avec déformation finie.*

L'hypothèse en question doit donc être admise, et c'est d'elle que les résultats demandés découlent. En particulier, elle montre immédiatement que l'élément linéaire du feuillet moyen déformé est approximativement celui d'un plan.

On en conclut que ce feuillet moyen affecte approximativement la forme d'une surface développable.

Mais il est clair d'après ce qui précède que cette déduction soulève une question d'analyse qu'il serait intéressant de résoudre.

Elle ne peut, en effet, s'opérer que par des différentiations. Du moins, toutes les méthodes connues par lesquelles on démontre qu'une surface applicable sur un plan est nécessairement développable conduisent a différentier les coefficients de l'élément linéaire.

Si ces coefficients ne sont qu'approximativement égaux à l'unité ou à zéro, et si cette approximation ne se conserve pas dans la dérivation, peut on compter néanmoins que les méthodes dont nous venons de parler fournissent un résultat approché? Il est permis d'en douter, et de se demander si, dans ces conditions, les résultats obtenus ne seront pas analogues à ceux qui se présentent lorsque la différentiation des égalités exactes n'est pas permise et qui, comme l'a montré M. Lebesgue, sont profondément différents des résultats classiques.

Toutefois, il est à remarquer qu'on peut arriver au but sans faire intervenir l'hypothèse de différentiation dans un cas, celui où il s'agit d'une déformation

être négligés, et cependant assez minces pour que les autres approximations faites dans ce qui précède sóient légitimes; mais, jusqu'ici, la théorie ne nous renseigne pas à cet égard.

D'autre part, nous avons supposé que les coefficients d'élasticité étaient tous du même ordre de grandeur. Dans le cas contraire, comme les rapports de certaines de ces coefficients vien draient dans quelques unes des calculs précédents. multiplier l'épaisseur de la plaque, il y aurait lieu de voir si quelques uns des résultats ne se trouveraient pas modifiés.

* Certaines parties des raisonnements précédents subsisteraient, même dans ce cas. Ainsi nous pourrions encore démontrer que les tensions verticales sont très petites, par rapport aux horizontales, et même en déduire l'expression de $\mathrm{g}_{x z}, \mathbf{g}_{y z}, \mathfrak{g}_{z}$ en fonction de $\mathfrak{g}_{x}, \mathfrak{g}_{y}, \mathbf{g}_{x y}$. 
infiniment petite, et où les composantes de déformation peuvent être réduites à leurs parties linéaires, soient, pour trois d'entre elles:

$$
\frac{\partial u}{\partial x}, \quad \frac{\partial v}{\partial y}, \quad \frac{\partial u}{\partial y}+\frac{\partial v}{\partial x} .
$$

Prenons, en effet, les équations

$$
\begin{gathered}
\frac{\partial u}{\partial x}=\theta \epsilon, \quad \frac{\partial v}{\partial y}=\theta \epsilon, \\
\frac{\partial u}{\partial y}+\frac{\partial v}{\partial x}=\theta \epsilon
\end{gathered}
$$

oú $\epsilon$ désigne indifféremment plusieurs quantités du même ordre de grandeur.

Les deux premières donnent évidemment

$$
u=f(y)+\theta \epsilon A, \quad v=\phi(x)+\theta \epsilon A
$$

$A$ étant la plus grande dimension de la plaque.

Il nous est impossible de différentier les deux équations précédentes pour obtenir les valeurs de $\partial u / \partial y, \partial v / \partial x$ afin de les transporter dans la relation (18). Nous intégrerons donc celle-ci daus l'aire d'un rectangle ayant ses côtés parallèles aux axes de coordonnées et représentés par les équations

Il viendra

$$
\begin{gathered}
x=x_{1}, \quad x=x_{2}, \quad y=y_{1}, \quad y=y_{2} . \\
\int(u d x-v d y)=\theta \epsilon\left(x_{2}-x_{1}\right)\left(y_{2}-y_{1}\right),
\end{gathered}
$$

ou, en tenant compte de (19),

$$
\begin{aligned}
&\left(x_{2}-x_{1}\right)[\left.f\left(y_{1}\right)-f\left(y_{2}\right)\right]-\left(y_{2}-y_{1}\right)\left[\phi\left(x_{2}\right)-\phi\left(x_{1}\right)\right] \\
&=\theta \epsilon\left(x_{2}-x_{1}\right)\left(y_{2}-y_{1}\right)+\theta \epsilon A\left(\left|x_{2}-x_{1}\right|+\left|y_{2}-y_{1}\right|\right) .
\end{aligned}
$$

Par un point $a$ du contour de la plaque, menons des parallèles $a b, a c$ aux axes, jusqu'à nouvelle rencontre en $b, c$ avec le contour (Fig. 2). La parallèle menée par le point $b$ à l'axe des $y$ coupera à nouveau le contour en $d$ et la parallèle menée par $c$ à l'axe des $x$, en $d^{\prime}$. Nous allons, pour simplifier, supposer

$1^{\circ}$ que la plaque est convexe;

$2^{\circ}$ qu'elle est entièrement comprise à l'intérieur de la figure formée par les rectangles $a b d c, a c d^{\prime} e^{\prime}$ (Fig. 2) et les demi cercles décrits extérieurement à ces rectangles, sur $a b, a c, c d, d^{\prime} b$ comme diamètres respectifs.

Soient $x_{0}, x_{0}+B, x_{0}+B^{\prime}$ les abscisses des points $a, d^{\prime}, d ; y_{0}, y_{0}+C^{\prime}$, $y_{0}+C$, les ordonnées de ces mêmes points, et posons

$$
\frac{\phi\left(x_{0}+B\right)-\phi\left(x_{0}\right)}{B}=r .
$$




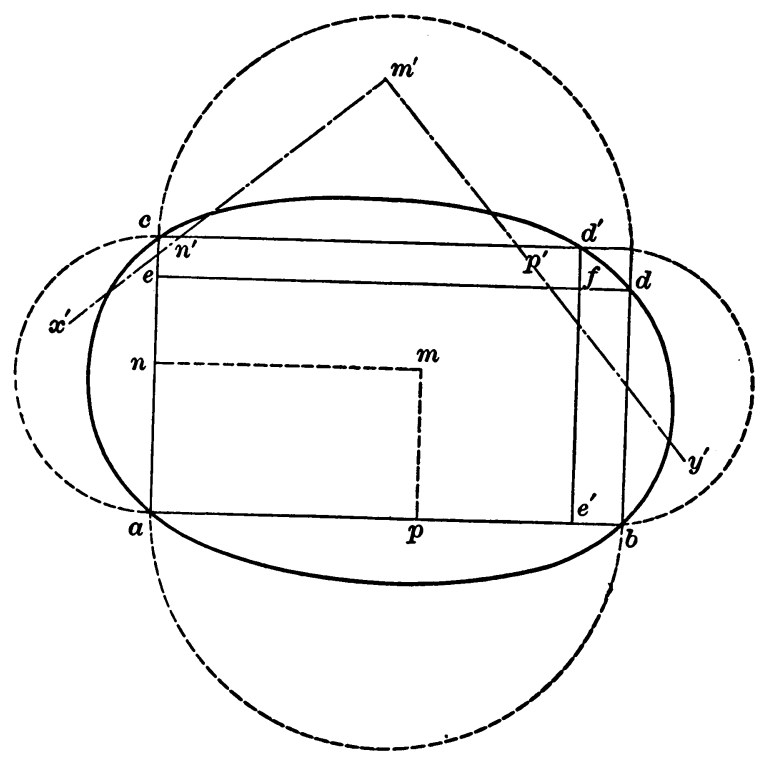

FIG. 2.

La relation (20), appliquée aux rectangles $a b d e, a c d^{\prime} e^{\prime}, a e e^{\prime} f$ nous donnera

$$
\begin{aligned}
& \frac{f\left(y_{0}+C^{\prime}\right)-f\left(y_{0}\right)}{C^{\prime}}=-r+\theta \epsilon+\theta \epsilon\left(\frac{A}{B}+\frac{A}{C^{\prime}}\right), \\
& \frac{f\left(y_{0}+C^{\prime}\right)-f\left(y_{0}\right)}{C}=-r+\theta \epsilon\left(\frac{A}{B}+\frac{A}{C}+1\right), \\
& \frac{\phi\left(x_{0}+B^{\prime}\right)-\phi\left(x_{0}\right)}{B^{\prime}}=r+\theta \epsilon\left(\frac{A}{B}+2 \frac{A}{C}+\frac{A}{B^{\prime}}+2\right) .
\end{aligned}
$$

$\mathrm{Si}$, de plus, $m$ est un point quelconque intérieur à l'un des deux rectangles, il sera à une distance supérieure à $B / 2$ de l'un au moins des côtés $a c, b d, d^{\prime} e^{\prime}$, et à une distance supérieure à $C / 2$ de l'un au moins des côtés $a b, d e, c^{\prime} d^{\prime}$.

La relation (20) appliquée à un rectangle tel que amnp (Fig. 2) nous donnera dès lors

$$
\begin{aligned}
& f(y)=f\left(y_{0}\right)-r\left(y-y_{0}\right), \\
& \phi(x)=\phi\left(x_{0}\right)+r\left(x-x_{0}\right),
\end{aligned}
$$

avec des erreurs qui seront dans un rapport fini avec $\epsilon$.

Considérons enfin un point $m^{\prime}$ extérieur à nos rectangles : compris, par exemple, dans le cercle décrit sur $c d$ comme diamètre. Par ce point, nous pourrons mener deux droites perpendiculaires entre elles et qui coupent toutes deux les rectangles. Soient $x^{\prime}, y^{\prime}$ des coordonnées rapportées à ces droites prises comme 
axes ; $u^{\prime}, v^{\prime}$ les projections des déplacements correspondants ; $\alpha$, l'angle de l'axe des $x^{\prime}$ avec l'axe des $x$. Les relations (17) donneront aisément

$$
\left|\frac{\partial u^{\prime}}{\partial x^{\prime}}\right|<\epsilon, \quad\left|\frac{\partial v^{\prime}}{\partial y^{\prime}}\right|<\epsilon .
$$

Mais nous supposons que l'axe des $x^{\prime}$ contient un point $n^{\prime}$ intérieur à nos rectangles,-pour lequel, on a par conséquent

$$
u=f\left(y_{0}\right)-r\left(y-y_{0}\right)-\epsilon D, \quad v=\phi\left(x_{0}\right)+r\left(x-x_{0}\right)+\epsilon D
$$

( $D$ étant fini) ce qui permettra d'obtenir des valeurs approchées pour la quantité $u^{\prime}$; - -et l'axe des $y^{\prime}$, un point $p^{\prime}$ pour lequel on pourra de même écrire une expression de $v^{\prime}$.

Des valeurs ainsi obtenues pour $u^{\prime}$ au point $n^{\prime}$ et pour $v^{\prime}$ au point $p^{\prime}$, les relations (21) permettent évidemment de déduire les valeurs approchées pour $u^{\prime}$ et $v^{\prime}$ (et, par conséquent, pour $u$ et $v$ ) en $m^{\prime}$.

Le résultat est évidemment que $u$ et $v$ sont, dans toute l'étendue de la plaque, de la forme

$D$ étant fini.

$$
\begin{aligned}
& u=f\left(y_{0}\right)-r\left(y-y_{0}\right)+\epsilon D, \\
& v=\phi\left(x_{0}\right)+r\left(x-x_{0}\right)+\epsilon D,
\end{aligned}
$$

C'est bien la forme que nous devions trouver pour les déplacements $u, v$.

\section{IX}

Nous terminerons par deux remarques d'ordre purement analytique.

La solution des difficultés présentées par la formation des conditions aux limites réside, nous l'avons rappelé, dans ce fait qu'il se produit des déformations notables au voisinage des bords et négligeables partout ailleurs : ces déformations complémentaires satisfont à l'équation

$$
\frac{\partial^{2} u}{\partial x^{2}}+\frac{\partial^{2} u}{\partial y^{2}}=k^{2} u
$$

où $k$ est très grand (de l'ordre de l'inverse de l'épaisseur).

Lorsque le domaine considéré est circulaire, on reconnait aisément que les solutions de cette équation diminuent très rapidement dès qu'on s'éloigne du contour.

On peut arriver à la même conclusion, dans le cas général, de la manière suivante.

Considérons une solution $u$ de l'équation (22), les valeurs absolues de $u$ sur le contour étant toutes inférieures à $M$. Soit $O$ un point, dont la distance minimum au contour soit $\delta$. L'équation (22) admettra la solution $J(i k r) / J(i k \delta)$, 
où $r$ est la distance d'un point quelconque $(x, y)$ au point $O$ et $J$ la fonction de Bessel.

Or, sur tout le contour, $r$ étant supérieur à $\delta$ et $|u|$ inférieur à $M$, les fonctions $u+M J(i k r) / J(i k \delta), u-M J(i k r) / J(i k \delta)$ seront, l'une positive, l'autre négative. Il en sera done de même à l'intérieur, et au point $O$, en particulier, l'on aura

$$
|u|<\frac{M}{J(i k \delta)},
$$

inégalité qui met bien en évidence la diminution rapide de $|u|$ dès que $\delta$ n'est plus très petit, si $k$ est très grand.

$\mathbf{X}$.

Le mode de croissance de la fonction $J(i x)$, pour $x$ positif et très grand est celui d'une exponentielle.

Plus généralement, on peut, ainsi qu'il est bien connu, trouver un développement qui représente asymptotiquement $J(i x)$ pour les grandes valeurs de $x$.

Mais ce développement n'est que formel. Prolongé indéfiniment, il est divergent pour toute valeur de la variable.

M. Borel a perfectionné, dans ces dernières années, l'ćtude des developpements de cette espèce. On pourrait chercher a déduire de ses travaux une expression de $J(i x)$ mettant en évidence son mode de croissance, qui soit cependant convergente.

Les considérations suivantes (tout analogues à une démonstration bien connue de Weierstrass) conduiraient peut-ĉtre au même résultat.

Soit la série de puissances

$$
a_{0}+a_{1} x+\cdots+a_{m} x^{m}+\cdots,
$$

divergente pour toute valeur de $x$ et que nous considérons pour $x$ r'éel et positif (ou du moins en supposant que l'argument de $x$ est compris entre deux limites suffisament voisines de zéro).

On a

$$
e^{\frac{1}{x}}=1+\frac{1}{x}+\frac{1}{2 !} \cdot \frac{1}{x^{2}}+\cdots+\frac{1}{m !} \cdot \frac{1}{x^{i n}}+\cdots
$$

Soit $P_{p}(1 / x)$ l'ensemble des premières termes de ce développement. La différence $1-e^{-1 / x} P_{p}(1 / x)$ est plus petite en valeur absolue qu'un nombre $\epsilon$, que l'on peut rendre aussi petit qu'on veut en prenant $p$ suffisament grand si $|x|$ est supérieur à un nombre donné $\alpha$.

Alors, en designant par $\alpha_{i}$ des nombres tendant vers zéro, par $a_{i} \epsilon_{i}$ les termes d'une sćrie absolument convergente, et par $p_{i}$ des entiers tels

$$
\left|1-e^{-\frac{1}{x}} P_{p_{i}}\left(\begin{array}{l}
1 \\
x
\end{array}\right)\right|
$$


soit inférieur à $\epsilon_{i}$ pour $|x|>\alpha_{i}$, la série

$$
a_{0}+a_{1} x\left[1-e^{-\frac{1}{x}} P_{p_{1}}\left(\frac{1}{x}\right)\right]+\cdots+a_{m} x^{m}\left[1-e^{-\frac{1}{x}} P_{p_{m}}\left(\frac{1}{x}\right)\right]+\cdots
$$

sera convergente et aura les mêmes valeurs asymptotiques que (23).

Cette méthode peut évidemment être variée d'une infinite de manières, en changeant les valeurs des entiers $p_{i}$ et en remplaçant $e^{-1 / x}$ par d'autres exponentielles. En changeant $x$ en $1 / x$ et appliquant à la série divergente qui intervient dans le calcul de $J(i x)$, on pourrait espérer obtenir une expression exacte de cette fonction. 